\title{
Mechanism of Deformation Twinning in Tantalum Driven by Extremely Dynamic Shear-compression at Low Temperature
}

\author{
C. Q. Chen ${ }^{1,2}$, J. N. Florando ${ }^{3}$, M. Kumar ${ }^{3}$, K. T. Ramesh ${ }^{1}$, K. J. Hemker ${ }^{1}$
}

${ }^{1}$. Department of Mechanical Engineering, and Hopkins Extreme Materials Institute, Johns Hopkins University, Baltimore, MD, USA

2. Department of Materials Science and Engineering, Northwestern University, Evanston, IL, USA

${ }^{3 .}$ Materials Engineering Division, Lawrence Livermore National Laboratory, Livermore, CA, USA

The nucleation and growth of deformation twins, and their relationship with dislocation slip in bcc metals have long been attracting considerable research attention, but have not been fully understood [1]. In this work, we successfully introduced deformation twinning in tantalum through low temperature shear compression experiments [2] (see Fig. 1 for specimen geometry), which allow dynamic testing under shear dominant mode at strain rates up to $\sim 10^{4} / \mathrm{s}$, one order higher than typical conventional methods.

Transmission electron microscopy (TEM) has successfully captured the early stage of the formation of deformation twins. A commonly observed feature in all specimens deformed at $77 \mathrm{~K}$ is the interspersed nanometer-sized twin islands, with sizes ranging from tens to a few hundred nanometers. These twin islands were found to be aligned well along certain directions and separated by untwined regions; see Fig. 2 for an example. As shown in Fig. 2, the twin islands at the early stage of their formation have rather irregular and complex shapes. Close examination of the individual twin islands however shows that each of the incipient twins has a particularly geometry and is bounded by small facets of twinmatrix interfaces, but does not yet seem to have a well-defined habit plane.

Deformation twinning was confirmed through diffraction analysis in Fig. 3. Efforts were made to determine the plane of terminations that serves as the interface between the twin islands and the matrix through performing a series tilting experiments and detailed surface trace analysis. The planes of termination were determined through bringing these facets to edge on condition when they show sharp contrasts. A stereographic projection containing relevant planes and poles (zone axes) which was used for guiding the tilting experiments and trace analysis is also shown in Fig. 3.

The TEM analysis revealed a multiple-faceted growth characteristic of the incipient twins following at least two $\{112\}$ planes in the $<11 \overline{1}>$ zone, which at the same time serve as bother the twinning and slip systems. A relationship between slip and twinning nucleation is established in terms of the coalescence of twinning dislocations and dissociation of emissary dislocations. And the early stage growth of an incipient twin is rationalized through a slip assisted double-cross-slip growth mechanism.

The authors acknowledge funding support from LLNL. 


\section{References}

[1] Christian JW, Mahajan S. Deformation twinning. Prog. Mater. Sci. 1995;39:1.

[2] Jia D, Ramesh KT. Exp Mech 2004;44:445

[3] Rittel D, Bhattacharyya A, Poon B, Zhao J. Materials Science and Engineering: A 2007;447:65.
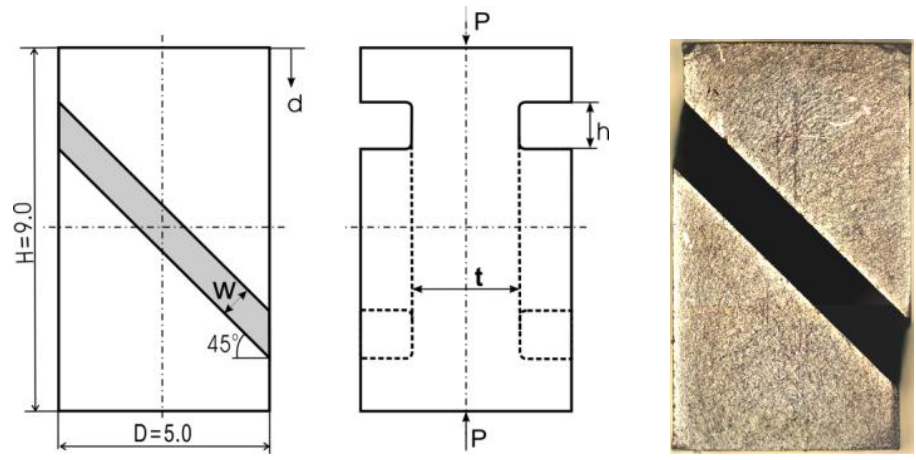

Figure. 1. Schematic representation of the shear compression specimen (left) and an image of a deformed shear compression specimen (right). Dimensions in the schematic are in millimeters.

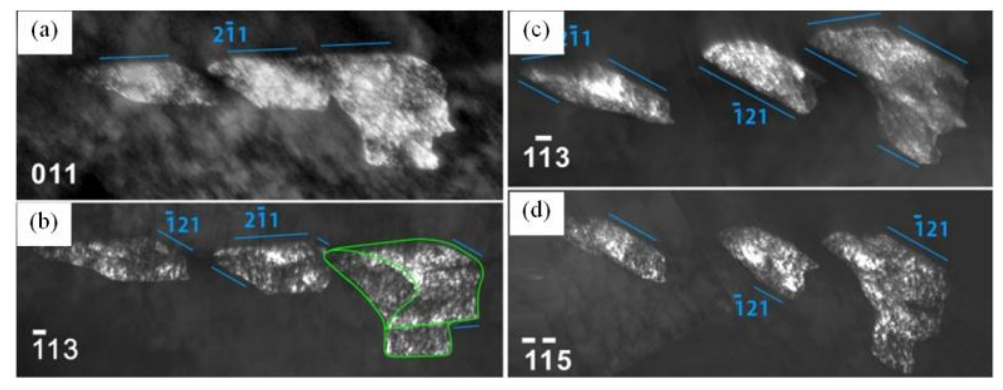

Figure 2. Detailed TEM study of the individual twins ahead of a grown-up twin: (a)-(d) TEM dark field (DF) images taken along different zone axes.
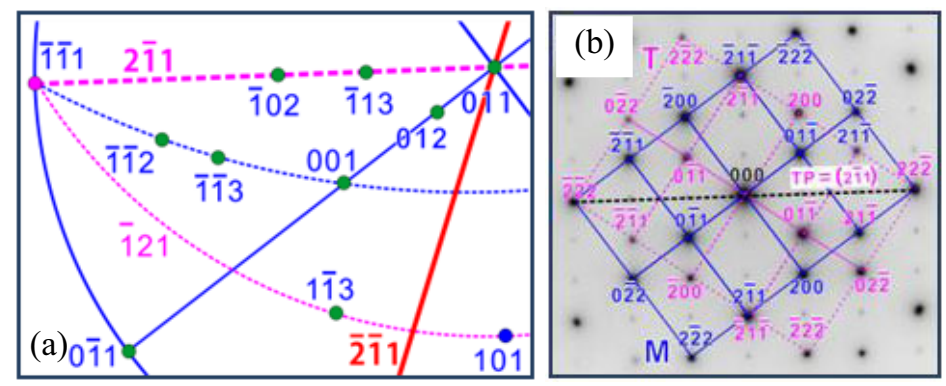

Figure 3. (a) [011] stereographic projection containing the zone axes used for observation in Fig. 2, (b) being composite SAED in [011] axis. 